\title{
Wannier Permanent Wave Functions for Featureless Bosonic Mott Insulators on the 1/3-Filled Kagome Lattice
}

\author{
S. A. Parameswaran, ${ }^{1}$ Itamar Kimchi, ${ }^{1}$ Ari M. Turner, ${ }^{2}$ D. M. Stamper-Kurn, ${ }^{1}$ and Ashvin Vishwanath ${ }^{1}$ \\ ${ }^{1}$ Department of Physics, University of California, Berkeley, California 94720, USA \\ ${ }^{2}$ Institute for Theoretical Physics, University of Amsterdam, Science Park 904, P.O. Box 94485, \\ 1090 GL Amsterdam, Netherlands
}

(Received 26 October 2012; published 19 March 2013)

\begin{abstract}
We study Bose-Hubbard models on tight-binding, non-Bravais lattices, with a filling of one boson per unit cell—and thus fractional site filling. We discuss situations where no classical bosonic insulator, which is a product state of particles on independent sites, is admitted. Nevertheless, we show that it is possible to construct a quantum Mott insulator of bosons if a trivial band insulator of fermions is possible at the same filling. The ground state wave function is simply a permanent of exponentially localized Wannier orbitals. Such a Wannier permanent wave function is featureless in that it respects all lattice symmetries and is the unique ground state of a parent Hamiltonian that we construct. Motivated by the recent experimental demonstration of a kagome optical lattice of bosons, we study this lattice at $1 / 3$ site filling. Previous approaches to this problem have invariably produced either broken-symmetry states or topological order. Surprisingly, we demonstrate that a featureless insulator is a possible alternative and is the exact ground state of a local Hamiltonian. We briefly comment on the experimental relevance of our results to ultracold atoms as well as to 1/3 magnetization plateaus for kagome spin models in an applied field.
\end{abstract}

DOI: 10.1103/PhysRevLett.110.125301

PACS numbers: $67.85 .-\mathrm{d}$, 05.30.Jp

Introduction.-Much recent activity in condensed matter physics has focused on identifying nontrivial phases of matter which are inherently quantum mechanical and cannot be understood by perturbing around a straightforward classical limit. Proposed systems where such phases might occur include strongly correlated electronic systems, frustrated antiferromagnets, and insulating phases of bosonic lattice systems, the last of which is our focus in this Letter. Unlike fermions, bosons are precluded from forming noninteracting band insulators, so all crystalline insulators require interactions, hence the term "Mott insulators" [1]. Mott insulators realized at integer fillings of bosons per site have a classical description deep within the insulating state in terms of a fixed integer number of particles per site, which means that, to find nontrivial states, fractional site filling is desirable.

Theoretically, it has been proven [2,3] that insulators at fractional filling per unit cell cannot be "featureless": They form either crystals with an enlarged unit cell by breaking lattice translation symmetry or exotic phases with topological order-i.e., phases with emergent excitations that carry unusual statistics. Experiments on cold atoms in optical lattices have extensively explored Mott insulating phases on a variety of simple lattices [4-7]. All these are Bravais lattices, with one site per unit cell —so that the site and unit cell fillings are identical-ruling out featureless states at fractional site filling.

However, recently, more complicated optical lattices with a basis—-such as the honeycomb [8] and kagome [9] structures-have been created, and the Mott and superfluid states in them have begun to be studied. This naturally leads us to consider fractional site filling, but integer unit cell filling. Are symmetry breaking or topological order still the only alternatives? We will study examples where the answer is no, and yet quantum fluctuations of bosons must be significant, even deep within a featureless insulating phase.

As an example of why a featureless Mott phase at fractional site filling can be counterintuitive, consider the kagome lattice at a filling of one boson per unit cell, or $1 / 3$ site filling. Symmetries conflict with the usual caricature of a Mott wave function, the essentially classical picture of a fixed number of bosons tied rigidly to each site. Attempting to draw such a classical cartoon on the kagome at the given filling leads inevitably to symmetry breaking, for example, a uniform $(q=0)$ state that distinguishes a single site in each triangle or the $\sqrt{3} \times \sqrt{3}$ order which enlarges the unit cell (Fig. 1). Arbitrarily choosing a unit cell, say, an upward-facing triangle, and delocalizing each boson across this choice of sites gives a more quantummechanical insulator but leads to a state which breaks point-group symmetries - specifically, in this "triangular" state, that of rotation by $180^{\circ}$ [10] (Fig. 1). Since the unit cell has three sites, which in the tight-binding limit are indivisible, one cannot write a state as a product of disjoint "molecules" while respecting the full sixfold point-group symmetry. Finally, more sophisticated approaches that first implement a duality transformation and then condense vortices of the dual order parameter also break symmetry at this filling [11]. Thus, for the kagome (and similar examples), fractional site filling ensures that there is no smooth connection to a trivial insulator. The usual 


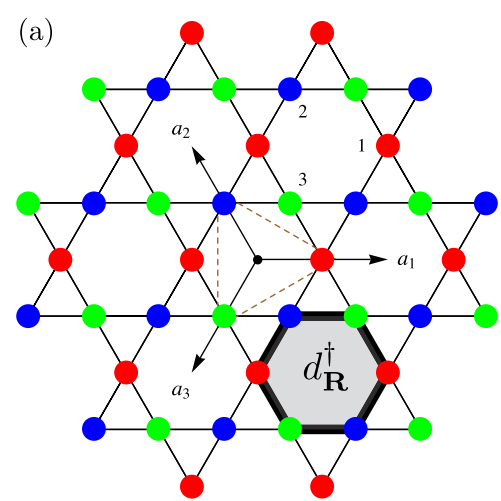

(b)

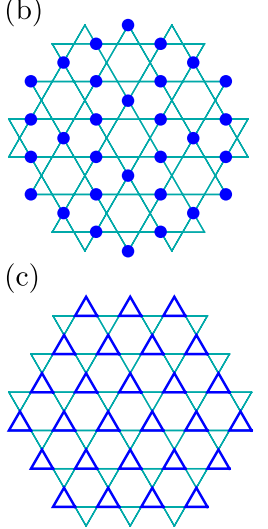

FIG. 1 (color online). (a) Kagome lattice and notation used in the text. The standard methods to construct featureless phases break symmetries, e.g., (b) the $\sqrt{3} \times \sqrt{3}$ state and (c) the triangular "molecular orbital" state.

strategies for constructing insulating phases always lead to broken symmetry, and to date no featureless insulating state has been found.

Here, we discuss a general scheme to write down wave functions and parent Hamiltonians for a class of such featureless quantum Mott insulators, including the 1/3-filled kagome as our primary example. Although we are interested in the problem of bosonic Mott insulators, we first study the reference problem of fermions at the same filling on the same lattice. If a band insulator of fermions exists, for which exponentially localized Wannier orbitals (WOs) that respect lattice symmetries can be constructed, then we show that a bosonic Mott insulating state is also feasible. The Mott insulating wave functions we construct are permanents [12] of these WOs-hence, we dub them Wannier permanent wave functions - and are analogous to fermionic band insulating states, which are determinants of the same orbitals. Furthermore, we demonstrate that these Wannier permanent wave functions are exact ground states of symmetric, local Hamiltonians of the Bose-Hubbard type. As an added bonus, the Bose-Hubbard models we discuss can also be viewed as describing $X X Z$ spin systems with fixed magnetization (e.g., in an applied field), for which our wave functions describe fractional magnetization plateaus which do not enlarge the unit cell. On the $1 / 3$-filled kagome lattice, we first find a tight-binding model which at this filling describes a band insulator and use this to construct a Wannier permanent wave function for the insulating phase. To the best of our knowledge, this is the first proposal of a featureless ground state for this problem.

Identifying a lattice like the kagome is subtle from a symmetry perspective: It entails taking the (experimentally well-motivated) tight-binding limit. Absent this restriction, the kagome, honeycomb, and triangular lattices are indistinguishable, as they all have the same space group. Formally, the tight-binding limit identifies a particular representation of the symmetry, which contains more

information than the space group. We can understand this intuitively: The kagome is a non-Bravais lattice with three sites per unit cell. Although solving a tight-binding model entails a specific choice of inequivalent sites to form the crystal basis (which breaks lattice symmetry), the final many-body band insulating wave function for fermions is independent of this choice and respects symmetries. In contrast, choosing orthogonal real-space orbitals to build a bosonic permanent is more sensitive; e.g., up- and downtriangle permanents are distinct, and both break $180^{\circ}$ rotation symmetry. Thus, restrictions placed by tight binding and the symmetrization of many-body bosonic wave functions make constructing featureless Bose insulators challenging, in contrast to the more usual case of fermionic band insulators.

The wave functions we study are in a sense bosonic analogs of the Affleck-Kennedy-Lieb-Tasaki states [13,14] of spin systems, which are quantum-mechanical paramagnets. As noted in Ref. [15], the Affleck-Kennedy-LiebTasaki insulators are "fragile" Mott insulators of electrons, which cannot be adiabatically connected to band insulators without breaking a crystalline point-group symmetry. Similarly, our wave functions cannot be deformed into disjoint product states without breaking translational or point-group symmetry. However, since we discuss bosons, in contrast to Ref. [15], we cannot consider a noninteracting limit, whatever the symmetry. We note previous work [16] that used power-law localized WOs to study Bose condensation and charge-density-wave order in the kagome flat band is unrelated to the featureless insulators we discuss.

Fermionic band insulators as bosonic Mott insulators.Given a set of symmetric, exponentially localized WOs $g_{\mathbf{R}}(i)$, with $\mathbf{R}$ a Bravais lattice vector and $i$ a lattice site, the Wannier permanent wave function for a bosonic Mott insulator takes the form $\left|\Psi_{W}\right\rangle=\prod_{\mathbf{R}} w_{\mathbf{R}}^{\dagger}|0\rangle$, where $w_{\mathbf{R}}^{\dagger} \equiv$ $\sum_{i} g_{\mathbf{R}}(i) b_{i}^{\dagger}$ adds a boson to the WO at $\mathbf{R} .\left|\Psi_{W}\right\rangle$ is the exact ground state of a parent Hamiltonian given by an interaction between densities projected into the WO basis. The correlation function can be expressed as a projector over the occupied band

$$
\left\langle b_{i}^{\dagger} b_{j}\right\rangle_{\Psi_{W}}=\sum_{\mathbf{R}} g_{\mathbf{R}}^{*}(i) g_{\mathbf{R}}(j)=\mathcal{P}_{\text {occ }}(j, i)
$$

and decays exponentially $\left\langle b_{i}^{\dagger} b_{j}\right\rangle \sim e^{-|i-j| / \xi}$, with $\xi$ related to the localization length of the WOs. Higherbody correlators can be similarly computed and also decay exponentially.

WOs are constructed by integrating Bloch wave functions over the Brillouin zone (BZ), with an arbitrary phase choice $\varphi_{\mathbf{k}}$ at each wave vector $\mathbf{k}$ [17]. For fermions, the fact that the different WOs are related to each other and to the single-particle Bloch eigenstates by unitary transformations means that "filling" any such complete set of single-particle states in the lowest band and then antisymmetrizing yields the same insulating wave function, as 
the resulting Slater determinants are all unitarily equivalent. However, symmetrized permanents such as $\left|\Psi_{W}\right\rangle$ are not unitarily equivalent for different choices of $g_{\mathbf{R}}(i)$, making the choice of single-particle states to fill crucial: Permanent wave functions for featureless Bose insulators must be built from localized and symmetric single-particle states.

To preserve symmetry, we must be able to fix the choice of localized WOs unambiguously. For a simple band-one that touches no other bands-respecting time reversal and inversion symmetry [18] fixes $\varphi_{\mathbf{k}}$ up to an overall sign, which is fixed by requiring continuity of $\varphi_{\mathbf{k}}$ across the BZ, a necessary condition for exponential localization [19-22]. Whenever a fermionic band insulator exists at a given filling, we can find such a set of WOs giving a symmetric insulating ground state and corresponding local parent Hamiltonian-a significant and hitherto unnoticed aspect of bosonic Mott physics.

Kagome lattice.-To use this approach for the kagome lattice at $1 / 3$ filling, we must find a fermionic band insulator (separated lowest band) on the kagome. We consider the general case $H_{0}\left[\left\{t_{i j}\right\}\right] \equiv-\sum_{i, j} t_{i j} b_{i}^{\dagger} b_{j}$ on the kagome lattice, where the $t_{i j}$ are assumed to respect all the symmetries of the lattice. $H_{0}$ has three bands, and, with only nearest-neighbor hopping $\left(t_{1}\right)$, the two lower bands form gapless Dirac points (Fig. 2). Mass terms that can gap out the Dirac points break inversion or threefold lattice symmetry (triangular and $\sqrt{3} \times \sqrt{3}$ states; see Fig. 1). However, as second- and third-neighbor hoppings $\left(t_{2}, t_{3}\right)$ are increased from zero, the bands become degenerate at $K, K^{\prime}$ when $t_{2}=t_{3}=\frac{1}{2} t_{1}$; thereafter, the lowest band is simple, with the twofold $K$-point degeneracy transferred to the upper two bands (see Fig. 2). (For a symmetry analysis,
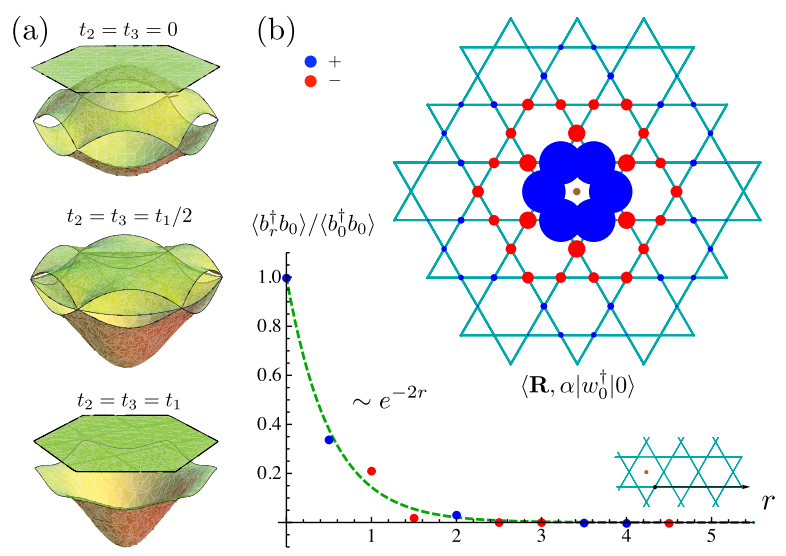

FIG. 2 (color online). (a) Evolution of the tight-binding band structure of $\mathrm{H}_{0}$; for the nearest-neighbor hopping (top), all bands touch, while, at the special point $t_{1}=t_{2}=t_{3}$ (bottom), the lowest band is isolated and is especially tractable. (b) WO plotted on the lattice. The size of the circles gives $\left|g_{\mathbf{R}}(i)\right|$; blue (red) circles depict $g_{\mathbf{R}}(i)>0(<0)$. The exponential decay of the boson correlator $\left\langle b_{r}^{\dagger} b_{0}\right\rangle$ is also shown below the WO. see Supplemental Material [23].) This procedure preserves lattice symmetry, providing symmetric WOs for the lowest band.

Wannier permanent wave functions.-For clarity, we focus initially on a fine-tuned point [24,25], where $t_{1}=$ $t_{2}=t_{3}=t / 6$ and the on-site potential $t_{i i}=t / 3$; our results persist away from this point as long as the lowest band remains simple. We may rewrite $H_{0}$ as $H_{0}=$ $-t \sum_{\mathbf{R}} d_{\mathbf{R}}^{\dagger} d_{\mathbf{R}}$ by defining operators that "smear" a boson over a single hexagon (Fig. 1 depicts $d_{\mathbf{R}}^{\dagger}$ for $\mathbf{R}=-\mathbf{a}_{2}$ ):

$$
d_{\mathbf{R}}^{\dagger} \equiv \frac{1}{\sqrt{6}} \sum_{\alpha=1,2,3}\left(b_{\mathbf{R}, \alpha}^{\dagger}+b_{\mathbf{R}-\mathbf{a}_{\alpha}, \alpha}^{\dagger}\right)
$$

Here, $\mathbf{R}=m \mathbf{a}_{1}+n \mathbf{a}_{2}$ lies on the triangular Bravais lattice; $\mathbf{a}_{1}, \mathbf{a}_{2}$, and $\mathbf{a}_{3} \equiv-\left(\mathbf{a}_{1}+\mathbf{a}_{2}\right)$ are shown in Fig. 1; and $\alpha=1,2,3$ labels inequivalent sites within a unit cell, so that a kagome site $i \equiv(\mathbf{R}, \alpha)$. Since $\left[d_{\mathbf{R}}, d_{\mathbf{R}^{\prime}}^{\dagger}\right] \neq \delta_{\mathbf{R}, \mathbf{R}^{\prime}}$, the $d_{\mathbf{R}}^{\dagger}$ operators are not canonical bosons and we cannot form a Fock space out of eigenstates of $d_{\mathbf{R}}^{\dagger} d_{\mathbf{R}}$. However, in momentum space, $H_{0}=-t \int_{\mathrm{BZ}} \frac{d^{2} q}{(2 \pi)^{2}} d_{\mathbf{q}}^{\dagger} d_{\mathbf{q}}$, where $d_{\mathbf{q}}^{\dagger}=$ $N_{s}^{-1 / 2} \sum_{\mathbf{R}} e^{-i \mathbf{q} \cdot \mathbf{R}} d_{\mathbf{R}}^{\dagger}$ with $N_{s}$ the number of sites. By a straightforward computation, $\left[d_{\mathbf{q}}, d_{\mathbf{q}^{\prime}}^{\dagger}\right]=\epsilon(\mathbf{q}) \delta_{\mathbf{q}, \mathbf{q}^{\prime}}$, with $\boldsymbol{\epsilon}(\mathbf{q})=1+\frac{1}{3} \sum_{i=1}^{3} \cos \left(\mathbf{q} \cdot \mathbf{a}_{i}\right)$. Since everywhere in the BZ $\epsilon(\mathbf{q}) \neq 0$, its inverse is nonsingular and we may define $\tilde{d}_{\mathbf{q}}^{\dagger} \equiv[\boldsymbol{\epsilon}(\mathbf{q})]^{-1 / 2} d_{\mathbf{q}}^{\dagger}$, so that $H_{0}=-t \int_{\mathrm{BZ}} \frac{d^{2} q}{(2 \pi)^{2}} \boldsymbol{\epsilon}(\mathbf{q}) \tilde{d}_{\mathbf{q}}^{\dagger} \tilde{d}_{\mathbf{q}}$, now in terms of canonical bosons $\left[\tilde{d}_{\mathbf{q}}, \tilde{d}_{\mathbf{q}^{\prime}}^{\dagger}\right]=\delta_{\mathbf{q}, \mathbf{q}^{\prime}} . H_{0}$ has a single band of dispersing states created by $\tilde{d}_{\mathbf{q}}^{\dagger}$ with energy $-t \epsilon(\mathbf{q})$ that is gapped away from $E=0$. In writing $H_{0}$ in this form, we have chosen a specific superposition of states in a unit cell and therefore a specific Bloch band. The two orthogonal states in the remaining Bloch bands form a pair of zero-energy flat bands (Fig. 2). $H_{0}$ respects all the kagome lattice symmetries, and its lowest band is manifestly simple, as discussed above.

A single boson is placed in the WO at $\mathbf{R}$ by the operator

$w_{\mathbf{R}}^{\dagger} \equiv \frac{1}{\sqrt{N_{s}}} \int_{\mathrm{BZ}} \frac{d^{2} q}{(2 \pi)^{2}} e^{i \mathbf{q} \cdot \mathbf{R}} \tilde{d}_{\mathbf{q}}^{\dagger}=\sum_{\mathbf{R}^{\prime}, \alpha} g_{\mathbf{R}^{\prime}}\left(\mathbf{R}^{\prime}, \alpha\right) b_{\mathbf{R}^{\prime}, \alpha}^{\dagger}$.

Here, the function

$$
g_{\mathbf{R}}\left(\mathbf{R}^{\prime}, \alpha\right)=\int_{\mathrm{BZ}} \frac{d^{2} q}{(2 \pi)^{2}} \frac{1+e^{i \mathbf{q} \cdot \mathbf{a}_{\alpha}}}{\sqrt{N_{s} \boldsymbol{\epsilon}(\mathbf{q})}} e^{i \mathbf{q} \cdot\left(\mathbf{R}^{\prime}-\mathbf{R}\right)}
$$

characterizes the spatial structure of the WOs (Fig. 2). It is easily verified from (3) that the $w_{\mathbf{R}}$ are canonical boson operators, i.e., $\left[w_{\mathbf{R}}, w_{\mathbf{R}^{\prime}}^{\dagger}\right]=\delta_{\mathbf{R}, \mathbf{R}^{\prime}}$. Filling one boson in each WO gives the Wannier permanent wave function [26].

Finally, each individual WO respects the point-group symmetry, as we can explicitly verify. Translations only map one WO into another, and therefore a product over all the WOs is invariant under the entire space group of the lattice, as is an arbitrary sum of such products, which 
includes a permanent wave function of the form $\left|\Psi_{W}\right\rangle$. Thus, we conclude that $\left|\Psi_{W}\right\rangle$ represents a featureless Mott insulator at filling 1/3 [27]. If we vary the parameters $t_{i j}$ away from the special point, the WOs will change. Nevertheless, as long as the WOs can be chosen to be symmetric and exponentially localized, the corresponding Wannier permanent wave function remains featureless and has exponentially decaying correlations. We thus have a family of Wannier permanent wave functions, each characterized by the hopping Hamiltonian $H_{0}$.

Parent Hamiltonian.-We construct a Hamiltonian for which the Wannier permanent wave function $\left|\Psi_{W}\right\rangle$ is an exact ground state. We do this by first constructing a new noninteracting Hamiltonian $H_{0}^{\prime}$, which flattens the dispersion of the lowest band while leaving its wave functions unaffected. Now, the WOs are actually single-particle eigenstates. We tune the chemical potential $\mu$ to be just above the lowest band. This leads to a massive ground state degeneracy, which is lifted by adding appropriate interaction terms that ensure the single occupancy of the WOs, thus selecting $\left|\Psi_{W}\right\rangle$ as the ground state.

We begin with the tight-binding problem $H_{0}$ and from it obtain the operators $w_{\mathbf{R}}^{\dagger}$. Using these, we construct a different single-particle Hamiltonian $H_{0}^{\prime}=-V \sum_{\mathbf{R}} w_{\mathbf{R}}^{\dagger} w_{\mathbf{R}}-$ $\mu \hat{N}$, where $\hat{N}$ is the total boson number. Using (3), $H_{0}^{\prime}$ can be expressed as a symmetric hopping model on the kagome lattice, whose locality follows from localization of the WOs. Matrix elements of $H_{0}^{\prime}$ decay exponentially (identically to $\left\langle b_{i}^{\dagger} b_{0}\right\rangle$, as shown in Fig. 2), and hopping between sites on nonadjacent hexagons is $\leqslant 3 \%$ of that between nearest neighbors. It is readily verified that $H_{0}^{\prime}$ may be rewritten as $H_{0}^{\prime}=-\sum_{\mathbf{R}}\left[(\mu+V) \hat{n}_{\mathbf{R}}^{w}+\mu\left(\hat{n}_{\mathbf{R}}^{u_{1}}+\right.\right.$ $\left.\hat{n}_{\mathbf{R}}^{u_{2}}\right)$ ], where $\hat{n}_{\mathbf{R}}^{w}=w_{\mathbf{R}}^{\dagger} w_{\mathbf{R}}$ represents the occupation of the WO of the lowest band at Bravais lattice site $\mathbf{R}$ and $\hat{n}_{\mathbf{R}}^{u_{1,2}}$ are occupations of the WOs of the two upper bands.

For $-V<\mu<0$ in $H_{0}^{\prime}$, only the $n_{\mathbf{R}}^{w}$ are nonzero. There are many ways to fill the WOs of the lowest band with bosons, leading to a huge degeneracy of many-body states. We lift this by adding to $H_{0}^{\prime}$ an interaction that penalizes multiple occupancy of a WO. This is accomplished by a term $H_{\text {int }}=\frac{U}{2} \sum_{\mathbf{R}} \hat{n}_{\mathbf{R}}^{w}\left(\hat{n}_{\mathbf{R}}^{w}-1\right)$. In terms of WOs, this is the familiar Hubbard interaction stabilizing a Mott phase with a fixed occupancy of each WO, but it corresponds to a more intricate interaction in terms of the site operators of the underlying kagome lattice [23]. Like $H_{0}^{\prime}, H_{\text {int }}$ is local in the sense that interactions between sites decay exponentially with their separation. It is easy to show that $\left|\Psi_{W}\right\rangle$ is the ground state of $H_{W} \equiv H_{0}^{\prime}+H_{\text {int }}$ for $-V<\mu<$ $\min (0, U-V)$ [28]. It is also clear that all excitations above the ground state are gapped. It is evident that $\left|\Psi_{W}\right\rangle$ is the unique state satisfying these properties on the torus, so it is not topologically ordered as it has no ground state degeneracy. In summary, we have shown that $\left|\Psi_{W}\right\rangle$ is the unique gapped and symmetric ground state of

$$
H_{W}=-V \sum_{\mathbf{R}} \hat{n}_{\mathbf{R}}^{w}+\frac{U}{2} \sum_{\mathbf{R}} \hat{n}_{\mathbf{R}}^{w}\left(\hat{n}_{\mathbf{R}}^{w}-1\right)-\mu \hat{N}
$$

for $-V<\mu<\min (0, U-V)$. An equivalent Hamiltonian can be obtained by first flattening the lowest band and then projecting a Hubbard interaction into it [29-31]. $H_{W}$ is intricate and presently challenging to realize experimentally (see below). Numerical study of simpler proximate Hamiltonians that may yield featureless phases is left to future work.

Experimental realization.-A natural experimental setting for Bose-Hubbard physics is in ultracold atomic gases in optical lattice potentials [4]. The kagome geometry was achieved recently in an experimental setup using an optical superlattice and was characterized by studying properties of an atomic superfluid with such a lattice [9]. Mott insulating states within this optical lattice have been recently observed [32].

Methods suitable for identifying an insulating state with fractional filling have been demonstrated for atoms in simple Bravais lattices. For example, Mott insulating states at various fillings can be identified by observing plateaus in the density of a lattice-trapped gas within an inhomogeneous potential $[6,7]$. The underlying geometry of the insulating state can be probed via momentum-resolved correlations in the measured atomic density [5], a method that can be employed to check against broken-symmetry states.

However, the fine tuning of next- and next-next-nearestneighbor hopping in such lattices may be difficult to achieve. One approach may be to utilize Feshbach resonances to increase the interaction strength so that a critical ratio of interaction to kinetic energies can be reached already in a very shallow optical lattice, where substantial higher-order tunneling may still occur. Alternately, returning to the analogy between bosonic tunneling and spin models, longer-range interactions can be achieved and tuned in lattice-trapped gases of polar molecules [33] or Rydberg atoms. Although $\left|\Psi_{W}\right\rangle$ is not sign positive, a related sign-positive wave function-potentially with better variational energies-exists in the same phase, albeit without a corresponding parent Hamiltonian [34].

We note that there are candidate materials where it is believed that the spin physics is captured by the isotropic kagome lattice Hamiltonian. By exploiting the connection between the Bose-Hubbard model and a spin system in an applied field, the wave functions we consider here are natural candidates for featureless fractional magnetization plateaus in such materials-particularly since their nontrivial sign structure suggests that they will give good variational energies [35].

Concluding remarks.-Although we have focused on a specific example of a non-Bravais Bose-Hubbard model, the kagome lattice at $1 / 3$ site filling, the path to generalizing our results is clear. For a lattice with a $q$-site unit cell, it is possible to construct a Wannier permanent at filling 
$1 / q$ if we can construct symmetric, exponentially localized WOs for the lowest band. The first step is to find a tightbinding model whose lowest band is simple, which often requires going beyond nearest-neighbor hopping, as the kagome teaches us [36]. In general, whether such a model exists depends on symmetries, as discussed above. For example, on the $1 / 2$-filled honeycomb lattice, the two bands that touch in the nearest-neighbor limit form a two-dimensional irreducible representation; as there is no third trivial band to which the degeneracy may be transferred, the touching cannot be removed without symmetry breaking [23], and, on nonsymmorphic lattices, featureless phases are impossible except at special integer fillings [37].

We note in closing that the kagome lattice WO is maximal on the hexagon of sites centered on Bravais lattice site $\mathbf{R}$ and decays rapidly away from it (Fig. 2). This suggests that the wave function $|\Psi\rangle=\prod_{\mathbf{R}} d_{\mathbf{R}}^{\dagger}|0\rangle$ obtained by filling "truncated" orbitals restricted to a hexagon remains in the same phase as $\left|\Psi_{W}\right\rangle$. Verifying this conjecture requires a numerical evaluation of correlations in the state $|\Psi\rangle$, which is a significant problem in its own right. One can by analogy construct a featureless insulating wave function on the honeycomb lattice, even though the Wannier construction fails $[23,34]$.

We thank M. Rypestøl, M. Zaletel, and an anonymous referee for comments on the manuscript, which contributed significantly to pedagogy. We acknowledge support from the Simons Foundation (S. A. P.), the NSF (I. K.), and the Army Research Office, with funding from the DARPA Optical Lattice Emulator program.

[1] M.P. A. Fisher, P. B. Weichman, G. Grinstein, and D. S. Fisher, Phys. Rev. B 40, 546 (1989).

[2] E. Lieb, T. Schultz, and D. Mattis, Ann. Phys. (N.Y.) 16, 407 (1961).

[3] M. B. Hastings, Phys. Rev. B 69, 104431 (2004).

[4] M. Greiner, O. Mandel, T. Esslinger, T. W. Hansch, and I. Bloch, Nature (London) 415, 39 (2002).

[5] S. Folling, F. Gerbier, A. Widera, O. Mandel, T. Gericke, and I. Bloch, Nature (London) 434, 481 (2005).

[6] S. Folling, A. Widera, T. Muller, F. Gerbier, and I. Bloch, Phys. Rev. Lett. 97, 060403 (2006).

[7] G. K. Campbell, J. Mun, M. Boyd, P. Medley, A.E. Leanhardt, L. G. Marcassa, D. E. Pritchard, and W. Ketterle, Science 313, 649 (2006).

[8] L. Tarruell, D. Greif, T. Uehlinger, G. Jotzu, and T. Esslinger, Nature (London) 483, 302 (2012).

[9] G.-B. Jo, J. Guzman, C. K. Thomas, P. Hosur, A. Vishwanath, and D. M. Stamper-Kurn, Phys. Rev. Lett. 108, 045305 (2012).

[10] L. Santos, M. A. Baranov, J. I. Cirac, H.-U. Everts, H. Fehrmann, and M. Lewenstein, Phys. Rev. Lett. 93, 030601 (2004).

[11] K. Sengupta, S. V. Isakov, and Y. B. Kim, Phys. Rev. B 73, 245103 (2006).
[12] The permanent of a square matrix $A_{i j}$ is defined as $\operatorname{perm}(A)=\sum_{\sigma \in S_{n}} \prod_{i=1}^{n} A_{i, \sigma(i)}$, where the sum extends over all elements $\sigma$ of the symmetric group $S_{n}$; i.e., it is analogous to a determinant without an alternating sign.

[13] I. Affleck, T. Kennedy, E. H. Lieb, and H. Tasaki, Phys. Rev. Lett. 59, 799 (1987).

[14] I. Affleck, T. Kennedy, E.H. Lieb, and H. Tasaki, Commun. Math. Phys. 115, 477 (1988).

[15] H. Yao and S. A. Kivelson, Phys. Rev. Lett. 105, 166402 (2010).

[16] S. D. Huber and E. Altman, Phys. Rev. B 82, 184502 (2010).

[17] Unitary transformations involving more than one band are allowed but unimportant here.

[18] Note that just requiring inversion symmetry forces a zero Chern number, so there is no topological obstruction to constructing WOs.

[19] J. Des Cloizeaux, Phys. Rev. 129, 554 (1963).

[20] J. Des Cloizeaux, Phys. Rev. 135, A685 (1964).

[21] J. Des Cloizeaux, Phys. Rev. 135, A698 (1964).

[22] W. Kohn, Phys. Rev. B 7, 4388 (1973).

[23] See Supplemental Material at http://link.aps.org/ supplemental/10.1103/PhysRevLett.110.125301 for (i) symmetry analysis of band touchings and (ii) details of the parent Hamiltonian.

[24] D. L. Bergman, C. Wu, and L. Balents, Phys. Rev. B 78, 125104 (2008).

[25] L. Balents, M. P. A. Fisher, and S. M. Girvin, Phys. Rev. B 65, 224412 (2002).

[26] The exponential decay of $g_{\mathbf{R}}(i)$ and hence the boson correlations follow from the analyticity of $1 / \sqrt{\epsilon(\mathbf{q})}$ in the BZ.

[27] Note that the permanent wave function is a product state of orthogonal WOs, albeit not a product over disjoint realspace units. Orthogonality ensures that the symmetry of a single WO rules out symmetry breaking in the thermodynamic limit.

[28] Since $n_{\mathbf{R}}^{u_{j}}=0$ for $-V<\mu<0$ and $H_{\text {int }}$ ensures that $n_{\mathbf{R}}^{w}=1$ for $-V<\mu<U-V$, we obtain $\left|\Psi_{W}\right\rangle$ upon symmetrization.

[29] T. Neupert, L. Santos, C. Chamon, and C. Mudry, Phys. Rev. Lett. 106, 236804 (2011).

[30] D. N. Sheng, Z.-C. Gu, K. Sun, and L. Sheng, Nat. Commun. 2, 389 (2011).

[31] E. Tang, J.-W. Mei, and X.-G. Wen, Phys. Rev. Lett. 106, 236802 (2011).

[32] D. M. Stamper-Kurn (unpublished).

[33] H. P. Büchler, E. Demler, M. Lukin, A. Micheli, N. Prokof'ev, G. Pupillo, and P. Zoller, Phys. Rev. Lett. 98, 060404 (2007).

[34] I. Kimchi, S. A. Parameswaran, A. M. Turner, F. Wang, and A. Vishwanath, arXiv:1207.0498.

[35] Permanent wave functions have applied to magnetization plateaus on the anisotropic triangular lattice by T. Tay and O. I. Motrunich, Phys. Rev. B 81, 165116 (2010).

[36] The special simplifying features of the kagome, e.g., flat bands when $t_{2}=t_{3}=0, t_{1}$ are inessential; as long as a simple band exists, the Wannier approach works and is hence general.

[37] S. A. Parameswaran, A. M. Turner, D. P. Arovas, and A. Vishwanath, arXiv:1212.0557. 